\title{
NEONATAL DERIVED MESENCHYMAL STEM CELLS CAN BE ISOLATED FROM HUMAN UMBILICAL CORD WHARTON'S JELLY BUT NOT FROM HUMAN UMBILICAL CORD BLOOD
}

\author{
T. Paras Singh', M. L. Sherpa², Anup Pradhan', T. A. Singh ${ }^{4}$
}

${ }_{1}^{1}$ Lecturer, Department of Biochemistry, Sikkim Manipal Institute of Medical Sciences, Sikkim Manipal University, Sikkim, India. 2 Professor, Department of Biochemistry, Sikkim Manipal Institute of Medical Sciences, Sikkim Manipal University, Sikkim, India. 3Professor, Department of Obstetrics and Gynaecology, Sikkim Manipal Institute of Medical Sciences, Sikkim Manipal University, Sikkim, India.

${ }^{4}$ Professor, Department of Biochemistry, Sikkim Manipal Institute of Medical Sciences, Sikkim Manipal University, Sikkim, India.

$$
\text { ABSTRACT }
$$

\section{BACKGROUND}

A successful isolation of Mesenchymal Stem Cells (MSCs) for its clinical application is very important. Isolation of MSCs from neonatal sources like human Umbilical Cord Wharton's Jelly (hUCWJ) have been reported in many studies and are considered primitive but isolation of MSCs from human umbilical cord blood (hUCB) is open to discussion.

\section{MATERIALS AND METHODS}

The MSCs from hUCWJ and hUCB were isolated by explant culture and Ficoll density gradient respectively. The media used for the two sources were Dulbecco's modified Eagles medium, GlutaMax and fetal bovine serum (FBS) for hUCWJ, and Iscove modified Dulbecco's medium and FBS for hUCB. Further, the isolated cells were studied as per criteria of International Society of Cellular Therapy to define the cells as MSCs. Finally, we compare the feasibility and ease of isolating the neonatal sources.

\section{RESULTS}

Unlike hUCWJ, defining hUCB cells to MSCs as per criteria is limited because the number of cells decreases once it reaches $50 \%$ confluence and after 2 weeks, they show differing morphology with an appearance of large dimension of multinucleated cells identifying them as osteoclast-like cells thus restricting further study. The cells derived from both the sources grew into long, spindle shaped cells with prominent nuclei but hUCB has limited proliferation and fulfilled only the first criterion to define as MSCs while the cells isolated from hUCWJ continue to grow for multiple passages and fulfil all the defining criteria.

\section{CONCLUSION}

Neonatal derived hUCWJ is more ideal source for isolation of MSCs than hUCB with the potential to use it in regenerative medicine.

\section{KEY WORDS}

Explant, Neonatal, Osteoclast-Like Cells, Stem Cells

HOW TO CITE THIS ARTICLE: Singh TP, Sherpa ML, Pradhan A, et al. Neonatal derived mesenchymal stem cells can be isolated from human umbilical cord Wharton's jelly but not from human umbilical cord blood. J. Evolution Med. Dent. Sci. 2019;8(12):849854, DOI: $10.14260 /$ jemds/2019/189

\section{BACKGROUND}

Embryonic and adult are the two major types of stem cells. Due to the ethical concerns, adult stem cells (ASC) are preferred over embryonic stem cells for research and therapy, even though the latter has a better capacity to regenerate and differentiate. The ASCs derived from different sites have different characteristics in terms of regeneration and differentiation. The Mesenchymal stem cells (MSCs) a subtype is emerging to be more popular than the others. MSCs was first isolated by Friedenstein et al in bone marrow ${ }^{1}$ which he differentiated it into adipocytes, osteocytes and chondrocytes. ${ }^{2}$

'Financial or Other Competing Interest': Dr. Singh reports grants from Indian Council of Medical Research (ICMR), other from Department of Biotechnology (DBT), during the conduct of the study.

Submission 03-01-2019, Peer Review 06-03-2019,

Acceptance 12-03-2019, Published 25-03-2019.

Corresponding Author:

T. Paras Singh,

Department of Biochemistry,

Sikkim Manipal Institute of Medical Sciences,

Sikkim Manipal University,

$5^{\text {th }}$ Mile Tadong, Gangtok,

Sikkim-737102, India.

E-mail: paras.s@smims.edu.in

DOI: $10.14260 /$ jemds/2019/189

\section{(c) (1) $(9)$}

Many researchers have isolated MSCs from different sources like adipose tissue, ${ }^{3}$ menstrual blood, ${ }^{4}$ amniotic fluid, ${ }^{5}$ umbilical cord, $, 6,7$ dental pulp 8 and placenta. ${ }^{9}$ MSCs also have unique properties of immune-modulation and homing i.e. it finds the site of injury and repairs it without any immune rejection. In animals, systemic administration of MSCs migrate to the injury site proving the migrant property of MSCs, but many researchers are unable to explain the proper mechanism behind it. It has been reported that homing of leukocytes in a specific tissue, the chemokines receptor with their ligands and adhesion molecules play an important role by implicating of hematopoietic precursors into and through the tissue. ${ }^{10,11}$ The deficient of immunogenicity in MSCs is due to the low expression of major histocompatibility complex - I and lack of costimulatory molecules such as CD40, CD80 and CD86. Further, they also have an effect on dendritic cell maturation by repression of $\mathrm{T}$ and $\mathrm{B}$ lymphocyte initiating and proliferation. ${ }^{12,13}$

A study on MSCs reported an age-related reduction in differentiating capacity with a possible decline in its therapeutic potential. ${ }^{14}$ While comparing younger cells and aged human-derived MSCs it has been observed that there is a decreased in proliferation rates, chondrogenic and osteogenic potential and also increased senescent features. ${ }^{15}$ 
Therefore, neonatal derived MSCs like Wharton jelly, placenta, umbilical cord blood, are considered primitive than those obtained from other tissues with higher proliferation and expansion potential. ${ }^{16}$ Chris $\mathrm{H}$. Jo et al in his study of multilineage differentiation by functional assays a large number of pluripotent stem cells markers like SOX2, Nanog, oct4, SSEA-4, TRA-1-81 can be detected during the expansion period thus proving the primitive nature. ${ }^{17}$

While comparing the enzymatic and explant technique, certain surface receptors digested from the extracellular matrix may transform the MSCs function by non-specific degradation, but explant culture does not required enzymes, therefore, evade this issue. MSCs from birth-associated tissues can be obtained in large quantities, and the required numbers of these stem cells can be transplanted in therapeutic approaches for tissue replacement.18 Neonatal derived MSCs have many benefits when compared to adults MSCs. First, neonatal MSCs have a significant expansion ability with rapid doubling time. Secondly, neonatal derived MSCs give rise to HLA-G but do not express class II human leukocytes antigens thus lacking immunogenicity. ${ }^{16,18}$

Human neonatal tissue provides an abundant source of developmentally young somatic cells that can be stored, differentiated and/or even reprogrammed for regenerative medicine. MSCs from neonatal tissue demonstrate greater proliferation capacity as compared to adult stem cells, and they are capable of differentiation into three germ layers, which confirms their pluripotency. ${ }^{19}$

The important issue of the neonatal derived MSCs is the availability of the sample compared to other adult stem cells. This study was conducted to compare the success rate of isolating MSCs derived from the two sources i.e. human umbilical cord blood (hUCB) and human umbilical Wharton's jelly (hUCWJ) in the laboratory of Sikkim Manipal Institute of Medical Sciences, Sikkim.

\section{MATERIALS AND METHODS}

This is an observational study in the Department of Biochemistry and Obstetrics and Gynaecology, Sikkim Manipal Institute of Medical Sciences. 15 samples of hUCB and 15 samples of hUCWJ were taken for the purpose of the study after due approval of the Institutional Ethics Board (SMIMS/IEC/C/2016-069) from the placental ends after Caesarean section. Informed consent of the mother was obtained from all participating mothers who have been screened for HIV, HBV and HCV.

\section{Isolation of MSCs from hUCB}

The hUCB were collected in a $50 \mathrm{ml}$ sterile tube containing $4 \mathrm{ml}$ of citrate-phosphate dextrose-A (CPD-A). Cord blood collections were performed under strict sterile conditions. The sample was processed within four hours of its collection. The cord blood was diluted to 1:1 ratio with phosphate buffer saline (PBS) for isolation of mononuclear cells (MNC). The diluted cord blood was carefully overlaid onto the FicollHypaque solution. Then it was centrifuged at $2000 \mathrm{rpm}$ for 35 min at room temperature. MNC were collected and washed with RBC lysis buffer for 5 minutes at three volumes to one and centrifuged at $7000 \mathrm{rpm}$ for 2 minutes. The supernatant was discarded, and the pellet washed with PBS at $200 \mathrm{~g}$ for 10 min, the process was repeated. Resuspend the cell in $5 \mathrm{ml}$ Iscove Modified Dubelco Medium (IMDM) supplemented with
$10 \% \mathrm{FBS}, 10 \mathrm{ng} / \mathrm{ml}$ human epidermal growth factor (HuEGF), $10 \mathrm{ng} / \mathrm{ml}$ human fibroblast growth factor (HuFGF) and 1\% antibiotic-antimycotic and transfer to $\mathrm{T} 25 \mathrm{~mm}$ culture flask and incubated at $37^{\circ} \mathrm{C}$ and $5 \% \mathrm{CO}_{2}$ for two days. Thereafter, change medium every three days.

\section{Isolation of hUCWJ MSCs}

Around $10 \mathrm{~cm}$ long hUCWJ was collected from the placental end in $50 \mathrm{ml}$ tube containing $32 \mathrm{ml}$ of Hank's Balance Salt Solution (HBSS) supplemented with $300 \mathrm{U} / \mathrm{ml}$ penicillin, 300 $\mu \mathrm{g} / \mathrm{ml}$ streptomycin, $150 \mu \mathrm{g} / \mathrm{ml}$ gentamicin and $1 \mu \mathrm{g} / \mathrm{ml}$ fungizone. Under a laminar flow hood, the UC was transferred to Petri dishes which contain PBS. It was washed 3-4 times to remove erythrocytes and blood clots. The cord was dissected vertically, and the umbilical vein and arteries removed. Chop the remaining part of the cord to make $2-3 \mathrm{~mm}^{2} /$ piece. Explants were transferred to a $35 \mathrm{~mm}$ culture plate and allowed to stand for $10 \mathrm{~min}$. Transfer $2 \mathrm{ml}$ DMEM GlutaMax containing $20 \%$ FBS and antibiotics. The culture plate was incubated at $37^{\circ} \mathrm{C}$ and $5 \% \mathrm{CO}_{2}$ for 4 days and the medium was changed every 3 days in 3 ml DMEM supplemented with $10 \%$ FBS and antibiotics. The obtained MSCs were characterized using the defining criteria as given below.

\section{In Vitro Differentiation}

Differentiation into Mesenchymal lineages like Osteoblast, Chondrocytes and Adipocytes under proper induction conditions is used to functionally ascertain "mesenchymalness" of MSCs according to Dominici et al and International Society for Cellular Therapy (ISCT) norms 20

\section{Adipogenic Differentiation}

The cell containing medium was aspirated from $60-80 \%$ confluence cells at the $3^{\text {rd }}$ passage and rinsed with $2 \mathrm{ml} \mathrm{PBS.} 2$ $\mathrm{ml}$ of adipogenic differentiation was added on to the culture plate. Incubated at $37^{\circ} \mathrm{C}$ and $5 \% \mathrm{CO}_{2}$ for 4 days. Re-fed culture every 3-4 days. Replaced medium with pre-warm adipogenic differentiation medium and continued incubation. MSCs continue to undergo limited expansion as they differentiated under adipogenic condition. After 14 days of cultivation, the adipogenic culture was stained with Oil Red 0 staining.

\section{Chondrogenic Differentiation}

The medium was aspirated from $60-80 \%$ confluence cell from $3^{\text {rd }}$ passage and rinsed with $2 \mathrm{ml}$ PBS. PBS was removed and 2 $\mathrm{ml}$ of trypsin was added to the culture plate and incubated for $7 \mathrm{~min}$ at $37^{\circ} \mathrm{C}$. The cell suspension was transferred to a $15 \mathrm{ml}$ centrifuge tube and pelleted at $1500 \mathrm{rpm}$ for $10 \mathrm{~min}$. 3-4 $5 \mu \mathrm{l}$ droplets were seeded on the culture plate to Generate micromass culture. After cultivating micromass cultures for 2 hrs, $2 \mathrm{ml}$ of prewarmed chondrogenic differentiation medium was added to the $35 \mathrm{~mm}$ culture plate and incubated at $37^{\circ} \mathrm{C}$ and $5 \% \mathrm{CO}_{2}$. The culture was re-fed with chondrogenic medium every 2-3 days. After 14 days of cultivation, chondrogenic pellets were stained with Alcian Blue Stain.

\section{Osteogenic Differentiation}

The cell containing medium was aspirated from $60-80 \%$ confluence cells from the $3^{\text {rd }}$ passage and rinsed with $2 \mathrm{ml}$ PBS. $2 \mathrm{ml}$ of osteogenic differentiation medium was added on to the culture plate. Incubated at $37^{\circ} \mathrm{C}$ and $5 \% \mathrm{CO}_{2}$ for 4 days. 
Re-fed culture every 3-4 days. Replaced medium with prewarmed osteogenic differentiation medium and continued incubation. MSCs continue to undergo limited expansion as they differentiated under osteogenic condition. After 21 days of cultivation, the osteogenic culture was stained with Alizarin Red S.

\section{Immunophenotyping by Flow Cytometry}

The ISCT has described a panel of markers which need to express by the adherent cell population, post tissue processing to label them as Mesenchymal stem cells. We used BD Stemflow Human MSC Analysis Kit where the following antibodies were provided, CD73-APC, CD105-PerCP-Cy5.5, CD90-FITC, CD44-PE and the PE channel open to use in combination with the supplied negative MSC cocktail (PE CD45, PE CD34, PE CD11b, PE CD19 and PE HLA-DR) and also included antibody conjugate.

\section{Statistical Analysis}

To examine the relation between the success of isolating MSCs from hUCWJ and hUCB, a statistical analysis was performed using Chi-square test of independence and $P$ value of $<0.05$ were considered statistically significant.

\section{RESULTS}

A high proliferation of MSCs is accountable for further characterization and therapeutic potential. This study compares the proliferating capacity of Mesenchymal stem cells derived from human neonatal tissue hUCWJ to that of hUCB. The "MSCs stemness" was determined by Dominici et al and ISCT for hUCWJ while that of hUCB was characterized morphologically, due to the limitation of cells numbers. The relation between these variables was significant, $\mathrm{X}^{2}(\mathrm{I})=$ $17.36, p=0.001$. hUCWJ demonstrated more success rate of isolating MSCs than by hUCB.

The mononuclear cells from the umbilical cord blood were isolated and the following observations were made. Out of the 15 cord blood samples, 4 were able to demonstrate features of fibroblastic cells which include the morphology and the plastic adherent property with a $27 \%$ success rate. These fibroblastic cells feature included the plastic adherent property and the morphology in the first passage. The isolation of mononuclear cells took an average of one and a half hour. Blood and debris were seen during P0 but is washed out in the subsequent medium change. It took 7 days on an average for the cells to appear as fibroblast spindle shape (fig. $1 \mathrm{~A}$ ) and by 14 days the cells were elongated, and colony formation was seen (fig. 1C). The cells reached $50 \%$ confluency in an average of 30 days and thereafter started dying. At around 10 days, cells that were morphologically similar to osteoblastic cells started appearing and the plate had a mix of both the fibroblastoid and the osteoblastic cells (fig. 2A \& 2B). Subsequent subcultures of the cells were not possible as proper numbers of the cell were not obtained to do further study thus fulfilling only the first criteria of Dominici et al.
The cells from hUCWJ were taken and the isolation process took approximately one hour to perform, which was faster than the mononuclear cell isolation. Migrations of cells from the explant hUCWJ were visible within 8-9 days of culture in the entire 15 samples giving $100 \%$ success rate (fig. 1B). These cells were uniformly longer and more fibroblast with prominent nuclei reaching $80-90 \%$ confluence within a week (fig.1D). The colony forming of hUCWJ was more distinct than the ones grown from cord blood. At about 15 days the explants are removed from the culture plate, the cells are trypsinized and split at 1:3 ratio. The cells grown form hUCWJ were sub cultured through a maximum of 19 passages successfully and thereafter showed signs of senescence.

The in vitro differentiation of MSCs into adipogenic differentiation was seen as early as 7 days of induction in adipogenic differentiation medium, the cells start showing the presence of lipid vacuoles under the microscope. After 14 days the cells were fixed with $4 \%$ formaldehyde and adipogenesis was confirmed by Oil Red 0 stain (Fig. 3A). For chondrogenic differentiation, the cells start aggregating within 2-3 days under chondrogenic differentiating medium. After 14 days of culture, a spheroid cell mass was formed which was fixed with $4 \%$ formaldehyde solution and stain with Alcian Blue. The blue staining indicated the synthesis of proteoglycans by chondrocytes (Fig. 3B). MSCs start showing mineralization under the microscope from day 10 under osteogenic differentiation medium. The MSCs were further cultured for 21 days and fixed with $4 \%$ formaldehyde. Calcium depositions were check using Alizarin Red S (Fig. 3C).

One more important characterization of MSCs to define as MSCs is Immunophenotypic by flowcytometry. The explant derived MSCs were analysed at 3 rd Passage which expresses positive markers CD73, CD105, CD90 and CD44 and lacks expression of negative MSC cocktail (PE CD45, PE CD34, PE CD11b, PE CD19 and PE HLA-DR) of all the cells population can be observed (Fig. 4).

This study also compared the ease of collecting and processing the sample where explant hUCWJ was found to be more cost-effective with higher success rates. The ease for processing hUCB increased with the addition of growth factor, like fibroblast growth factor (FGF) and Epidermal growth factor (EGF). Different studies have established the hUCB derived MSCs have to be processed within 6 hrs of collecting the sample and the proliferating capacity of MSCs decreases with increase in time, whereas for hUCWJ the success rates are high if processed within $24 \mathrm{hrs}$. During processing also, the hUCB has to go through many centrifugation processes (MNC, PBS wash etc) which actually increase the chance of contamination which is not in the case of hUCWJ. Therefore, hUCWJ explant culture is an easy, effective and feasible when compare to hUCB for the source of MSCs. 

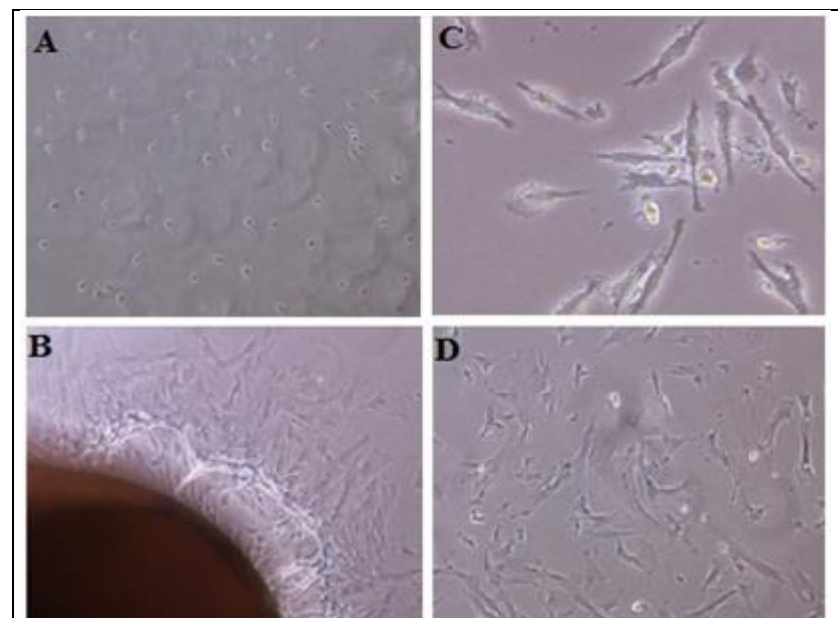

Figure 1. A. Spindle Shape Starts Appearing of CB Culture (Day 6). B. Cell Migration from the Ex-Plant (Day 9). C. Fibroblastoid Spindle Shape of hUCB MSCs (Day 10). D. hUCWJ Fibroblastoid Spindle Shape of MSCs After First Passage

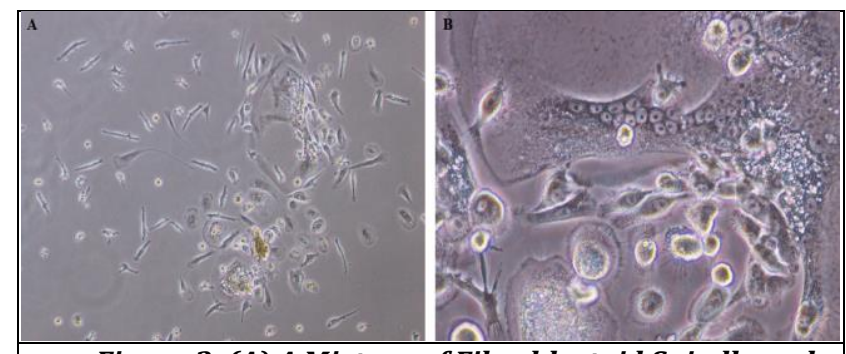

Figure 2. (A) A Mixture of Fibroblastoid Spindle and Osteoclast-Like Cells in 10X. (B) Fibroblastoid Spindle Surrounding Osteoclast-Like Cells 40X

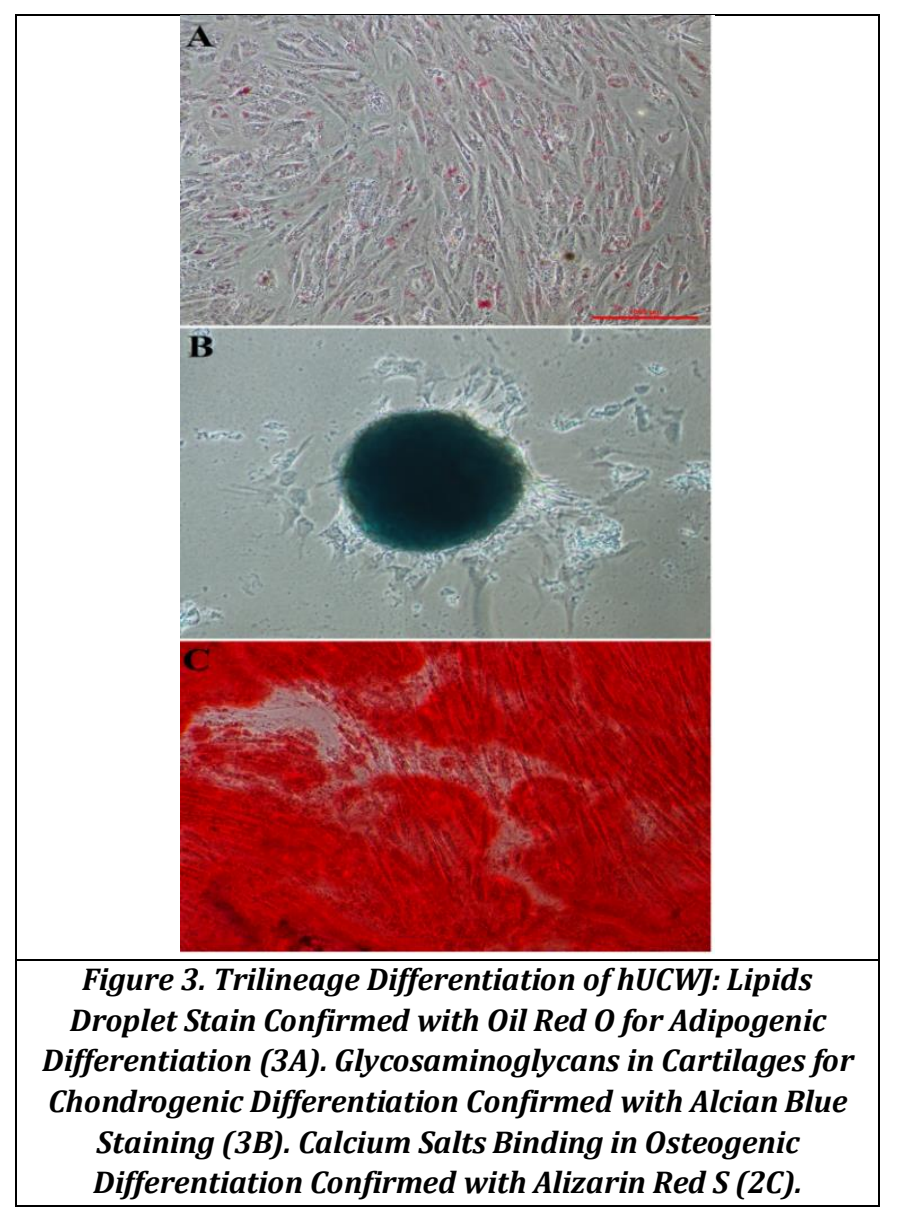

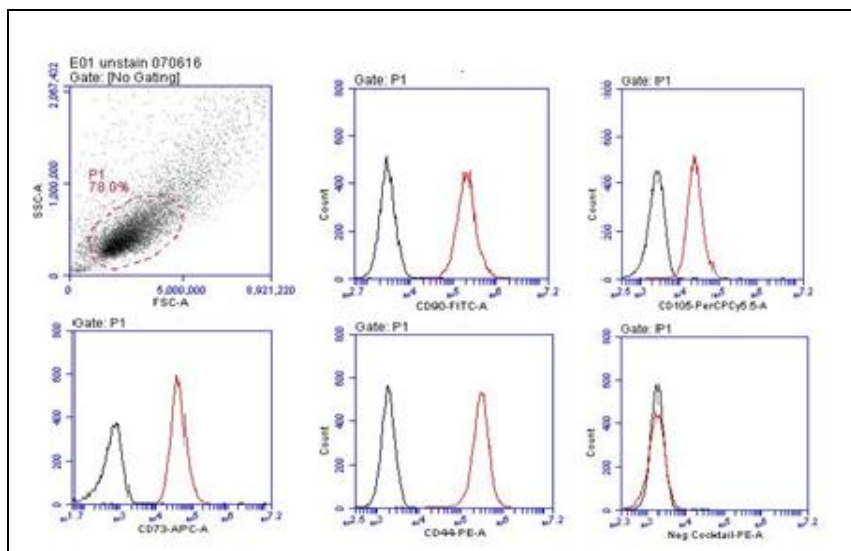

Figure 4. Expression of the Cell Surface Markers (CD90, CD105, CD73, CD44) and Lack of Expression of Negative Cocktails (PE CD45, PE CD34, PE CD11b, PE CD19 and PE HLA-DR) (Red Lines) when Compared with the Positive and Negative Isotype Control Cocktails (Black Lines). The Plots were Derived from Gated Events Based on the Light Scattering Characteristics of MSCs at Passage 3. Cells Were Analysed Using BD Accuri C6 Flow Cytometer

\section{DISCUSSION}

The main aim of the study was to compare the hUCB and hUCWJ as sources of MSCs. Out of all the sources of MSCs neonate derived MSCs are multipotent and can be differentiated into adipogenic, osteogenic, chondrogenic, myogenic and even endothelial lineages. ${ }^{21}$ But in our study, the hUCB derived cells fulfil only the first criteria for defining as MSCs. Our study findings are similar to the report of Erices A et al who reported that CB derived cells are adherent, and they show different morphology with the appearance of a high proportion of giant multinucleated cells after 2 weeks which were identified as osteoclast-like cells, previously reported.22

These osteoclasts were reported to have been derived from monocytes/macrophage precursor cells. Macrophage colony-stimulating factor (M-CSF) and receptor activator of nuclear factor- $\kappa \mathrm{B}$ ligand are critical differentiation factors of osteoclast. ${ }^{23}$ But osteoclast-like cells occurs in the culture is not possible without any cytokine-supplements. Minjun Yu et al suspect that this may be due to cell-cell interaction after confluence because no osteoclast-like cells were observed prior to culture in low density ${ }^{24}$ which show a similar result to our study as the osteoclast-like cells were not seen during the initial platting but started to appear once it reaches around $40 \%$ confluence.

The separation of fibroblastoid cells with MSCs from hUCB is contradictory. In our study, the hUCB were processed within $6 \mathrm{hrs}$ of collection and in an average of $30 \mathrm{ml}$ were collected for the isolation of MNC. But differing to our study Malgorzata Witkowska-Zimny et al reported in their study of full term UCB reported a greater yield of more than $60 \%$ of MSCs. They process the cord blood sample within 15 hours of the collection with a sample volume of more than $33 \mathrm{ml}$ and mononuclear count of more than $1 \mathrm{X}^{10} 0^{8}$. On the other hand, other authors have denied the presence of MSCs in hUCB and claimed that only hematopoietic cells can be isolated from hUCB. ${ }^{19}$ However, Friedenstein AJ et al suggested that FCS coated culture vessel enhance growth and attachment factors for MSCs derived from UCB. Thus, enable to remove the young myeloid and osteoclast-like cells and favouring the 
generation of MSC-like cells from hUCB. ${ }^{2}$ The frequency of hUCB derived MSC like cells were extremely low compared to the Bone marrow (BM). On the other hand, the clonal derived MSC like cells were able to generate much more progeny than the BM derived culture. 25

Karen Bieback et al reported that UCB from full term normal pregnancies not lasting over 37 weeks can give higher number of MSCs. They also reported that the duration of the storage time should not be more than 6 hours and not less than $80 \mathrm{ml}$ of umbilical cord blood. Kang et al also gave a similar report that they could not isolate MSCs from umbilical cord blood if the samples were collected and maintained for more than 6 hours. However, the role of FBS concentration and effects of supplements on media were not tested during the study.26,27

It has also been suggested that human articular chondrocytes (hACs) and human umbilical cord bloodderived Mesenchymal stem cells (hUCB-MSC) indirectly cocultured at low density has the potential to induce hUCBMSC differentiation into hyaline chondrocytes where TGF- $\beta 1$ may play a role in direct and SOX 9 may involve in indirect coculture system that induces hUCB-MSCs into chondrocytes. ${ }^{24,28}$

\section{CONCLUSION}

Our study corroborates the findings of researchers who have seen the low yield of mesenchymal stem cells (MSCs) in human umbilical cord blood (hUCB). We conclude that human umbilical cord Wharton's jelly (hUCWJ) is a superior source for getting high yield Mesenchymal stem cells. It also fits the defining criteria with relative ease to obtain and isolate in laboratories.

\section{Compliance with Ethical Standards and Funding}

We would like to thank the Indian Council of Medical Research (ICMR) for the funding (ICMR No: 5/7/1268/2015RCH). And, we would also like to thank IL Biotech Hub Project (Sanction No. 102/IFD/SAN/5881/2010-11), NER-BPMC, and Department of Biotechnology (DBT) Government of India, for its support.

\section{Conflict of Interest}

The authors declare that there is no conflict of interest.

\section{Ethical Approval}

Studies involving human participants were in accordance with the standards of the Institutional Ethical Committee. (SMIMS/IEC/C/2016-069).

\section{Informed Consent}

Informed consent was obtained from all participating mothers in the study.

\section{REFERENCES}

[1] Friedenstein AJ, Piatetzky-Shapiro II, Petrakova KV Osteogenesis in transplants of bone marrow cells. J Embryol Exp Morphol 1966;16(3):381-90.

[2] Friedenstein AJ, Deriglasova UF, Kulagina NN, et al. Precursors for fibroblasts in different populations of hematopoietic cells as detected by the in vitro colony assay method. Exp Hematol 1974;2(2):83-92.
[3] Bunnell BA, Flaat M, Gagliardi C, et al. Adipose-derived stem cells: isolation, expansion and differentiation. Methods 2008;45(2):115-20.

[4] Ulrich D, Muralitharan R, Gargett CE. Toward the use of endometrial and menstrual blood mesenchymal stem cells for cell-based therapies. Expert Opin Biol Ther 2013;13(10):1387-400.

[5] Roubelakis MG, Pappa KI, Bitsika V, et al. Molecular and proteomic characterization of human mesenchymal stem cells derived from amniotic fluid: comparison to bone marrow mesenchymal stem cells. Stem Cells Dev 2007;16(6):931-52.

[6] Romanov YA, Svintsitskaya VA, Smirnov VN. Searching for alternative sources of postnatal human mesenchymal stem cells: candidate MSC-like cells from umbilical cord. Stem Cells 2003;21(1):105-10.

[7] Wang HS, Hung SC, Peng ST, et al. Mesenchymal stem cells in the Wharton's jelly of the human umbilical cord. Stem Cells 2004;22(7):1330-7.

[8] Perry BC, Zhou D, Wu X, et al. Collection, cryopreservation and characterization of human dental pulp-derived mesenchymal stem cells for banking and clinical use. Tissue Eng Part C Methods 2008;14(2):149-50.

[9] In 't Anker PS, Scherjon SA, Kleijburg-van der Keur C, et al. Isolation of mesenchymal stem cells of foetal or maternal origin from human placenta. Stem Cells 2004;22(7):1338-45.

[10] Chamberlain G, Fox J, Ashton B, et al. Concise review: mesenchymal stem cells: their phenotype, differentiation capacity, immunological features and potential for homing. Stem Cells 2007;25(11):273949.

[11] Abdi R, Fiorina P, Adra CN, et al. Immunomodulation by mesenchymal stem cells: a potential therapeutic strategy for type 1 diabetes. Diabetes 2008;57(7):1759-67.

[12] Herrero C, Pérez-Simón JA. Immunomodulatory effect of mesenchymal stem cells. Braz J Med Biol Res 2010;43(5):425-30.

[13] Yunchuan D, Danmei X, Gang F, et al. Mesenchymal stem cell prevent the rejection of fully allogenic islet grafts by the immunosuppressive activity of matrix metalloproteinase-2 and -9 Diabetes 2009;58(8):1797-806.

[14] Yu JM, Wu X, Gimble JM, et al. Age-related changes in mesenchymal stem cells derived from rhesus macaque bone marrow. Aging Cell 2011;10(1):66-79.

[15] Marędziak M, Marycz K, Tomaszewski KA, et al. The influence of aging on the regenerative potential of human adipose derived mesenchymal stem cells. Article ID 2152435, Stem Cells International 2016;2016: p. 15.

[16] Troyer DL, Weiss ML. Wharton's Jelly-derived cells are a primitive stromal cell population. Stem Cells 2008;26(3):591-9.

[17] Jo CH, Kim OS, Park EY, et al. Foetal mesenchymal stem cells derived from human umbilical cord sustain primitive characteristics during extensive expansion. Cell Tissue Res 2008;334(3):423-33. 
[18] Hass R, Kasper C, Böhm S, et al. Different populations and sources of human mesenchymal stem cells (MSC): a comparison of adult and neonatal tissue-derived MSC. Cell Commun Signal 2011;9:12.

[19] Witkowska-Zimny M, Wrobel E. Perinatal sources of mesenchymal stem cells. Wharton's Jelly, amnion and chorion. Cell Mol Biol Lett 2011;16(3):493-514.

[20] Dominici M, Le Blanc K, Mueller I, et al. Minimal criteria for defining multipotent mesenchymal stromal cells. The International Society for Cellular Therapy position statement. Cytotherapy 2006;8(4):315-7.

[21] Vasaghi A, Dehghani A, Khademalhosseini Z, et al. Parameters that influence the isolation of multipotent mesenchymal stromal cells from human umbilical cord blood. Hematol Oncol Stem Cell Ther 2013;6(1):1-8.

[22] Erices A, Conget P, Minguell JJ. Mesenchymal progenitor cells in human umbilical cord blood. $\mathrm{Br} \mathrm{J}$ Haematol 2000;109(1):235-42.

[23] Miyamoto T, Ohneda O, Arai F, et al. Bifurcation of osteoclasts and dendritic cells from common progenitors. Blood 2001;98(8):2544-54.
[24] Yu M, Xiao Z, Shen L et al. Mid-trimester foetal bloodderived adherent cells share characteristics similar to mesenchymal stem cells but full-term umbilical cord blood does not. Br J Haematol 2004;124(5):666-75.

[25] Bieback K, Kern S, Klüter H, et al. Critical parameters for the isolation of mesenchymal stem cells from umbilical cord blood. Stem Cells 2004;22(4):625-34.

[26] Sibov TT, Severino P, Marti LC, et al. Mesenchymal stem cells from umbilical cord blood: parameters for isolation, characterization and adipogenic differentiation. Cytotechnology 2012;64(5):511-21.

[27] Kang XQ, Zang WJ, Bao LJ, et al. Differentiating characterization of human umbilical cord bloodderived mesenchymal stem cells in vitro. Cell Biol Int 2006;30(7):569-75.

[28] Li X, Duan L, Liang Y, et al. Human umbilical cord blood-derived mesenchymal stem cells contribute to chondrogenesis in coculture with chondrocytes. Article ID 3827057, Biomed Res Int 2016;2016: p. 9. 